\title{
GLAD!
}

Revue sur le langage, le genre, les sexualités

09 | 2020

Traductologies féministes

\section{Traduire He-Yin Zhen, perspectives sur la circulation des idées féministes}

Translating He-Yin Zhen: Perspectives on the Circulation of Feminist Ideas

\section{Léa Buatois}

\section{(2) OpenEdition}

1 Journals

Édition électronique

URL : http://journals.openedition.org/glad/2156

DOI : $10.4000 /$ glad.2156

ISSN : 2551-0819

Éditeur

Association GSL

Référence électronique

Léa Buatois, «Traduire He-Yin Zhen, perspectives sur la circulation des idées féministes », GLAD! [En ligne], 09 | 2020, mis en ligne le 20 décembre 2020, consulté le 20 janvier 2021. URL : http:// journals.openedition.org/glad/2156; DOI : https://doi.org/10.4000/glad.2156

Ce document a été généré automatiquement le 20 janvier 2021.

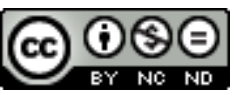

La revue GLAD! est mise à disposition selon les termes de la Licence Creative Commons Attribution Pas d'Utilisation Commerciale - Pas de Modification 4.0 International. 


\title{
Traduire He-Yin Zhen, perspectives sur la circulation des idées féministes
}

Translating He-Yin Zhen: Perspectives on the Circulation of Feminist Ideas

\author{
Léa Buatois
}

1 Cet article propose une réflexion sur les enjeux de la traduction des textes de He-Yin Zhen 何殷震 (1884-v.1920), anarchiste et féministe chinoise, mais souhaiterait aussi dépasser ce cadre pour esquisser des questionnements sur les études chinoises et la traduction féministe en général. Il est issu d'une intervention proposée en février 2019 au sein d'un séminaire de traductologie féministe du projet FELiCiTE (Féminismes en Ligne: Circulations, Traductions, Éditions), et présente une première étape de recherches en cours.

Originaire de Yangzhou, He-Yin Zhen grandit dans une famille aisée où elle reçoit une éducation classique rigoureuse. On a relativement peu d'informations sur son enfance et son adolescence. Elle épouse Liu Shipei 劉師培 (1884-1919) en juin 1904 et rejoint Shanghai en juillet. Elle entre alors au Centre d'études patriotiques [révolutionnaires] pour les femmes (Aiguo nüxue 愛國女學,fondé en 1902 par l'intellectuel Cai Yuanpei 蔡 元培, 1868-1960), où elle lit de la théorie anarchiste en mandarin et en japonais, et fait la rencontre de nombreux'ses intellectuellle's révolutionnaires, dont certain'e's qu'elle retrouvera plus tard jusqu'au Japon, comme son amie Tong Nutao 同怒濤 (dates inconnues). Elle commence la même année à écrire des articles dans des journaux révolutionnaires comme le Jingzhong ribao 警鐘日報 où elle écrit un court poème en hommage à Lin Zongsu 林宗素 (Wan $2011: 2$ ).

3 Suite à la répression de leurs activités par les autorités, elle décide de se rendre à Tokyo en février 1907 avec son compagnon Liu Shipei et d'autres ami-e's. He-Yin Zhen y met en place, aux côtés des militantes chinoises Lu Huiquan 陸恢權, Xu Yazun 徐亞尊 et Tong Nutao 同怒濤, l'Association pour la restauration des droits des femmes (Nüzi fuquan hui 女子復權會) en juin 1907, et fonde le même mois le journal de la Justice 
Naturelle (Tianyi 天義) avec Liu Shipei. Tokyo est historiquement un centre important des textes féministes en langue chinoise, car de nombreuses filles y sont allées compléter et poursuivre leurs études dans les premières écoles de filles, encore rares en Chine. C'était aussi un foyer bouillonnant de traductions et de discussions sur l'anarchisme et le socialisme (Liu $2017: 3$ ).

Iels s'entourent de nombreux'ses militante's anarchistes, communistes et socialistes, comme Zhang Taiyan 章太炎 (1869-1936) et le Japonais Kotoku Shusui 幸德秋水 (1871-1911), avec qui iels participent au Groupe de lecture et d'étude du socialisme (Shehui zhuyi jiangxi hui 社會主義講習會). L'objectif de ce groupe était, selon elleux, de permettre aux Chinoisees de Tokyo d'apprendre davantage sur les théories anarchocommunistes, et de discuter les limites des discours nationalistes qui avaient alors un succès considérable (Liu $2017: 7$ ).

Elle prend la parole pendant la première réunion de ce groupe en août 1907, pour présenter son journal aux autres participante-s, en expliquant qu'il a pour but de diffuser des idées relatives à l'égalité entre les hommes et les femmes, et à l'anarchisme (He-yin b : 310). Dans ses articles, elle explique pourquoi, de la tradition confucéenne au système capitaliste et aux politiques parlementaires, tout est fondé sur l'exploitation des classes et des sexes. Elle montre pourquoi un processus révolutionnaire qui ne serait pas mené par les femmes elles-mêmes est voué à l'échec.

Dans «l'Annonce concernant le journal Justice Naturelle», elle explique que le journal a été créé pour être «l'outil » de l'Association pour la restauration des droits des femmes (He-Yin a : 137). Elle écrit : «chaque numéro sera composé d'une vingtaine de pages, avec des illustrations, des discussions de société, des articles théoriques, des commentaires d'actualité, des traductions, des contributions, des textes divers (zaji 雜 記), avec pour objectif d'éveiller le monde et le peuple» (TY : 580). Ainsi est décrit le choix du titre du journal, dans les «Principes [de publication]» du premier numéro, rédigé avec d'autres contributeurices :

Afin de détruire la société, et de réaliser notre objectif qui est celui de l'égalité parmi tous les êtres humains, nous promouvons d'une part la révolution des femmes (nüjie geming 女子革命), mais aussi la révolution raciale (zhongzu geming 種 族革命), politique et économique, ce qui donne le nom de tianyi [justice naturelle] au journal (TY : 580).

7 Tianyi est un journal bimensuel, édité entre juin 1907 et juin 1908, avec un total de dixneuf numéros. Par rapport à la Revue des Nouvelles Femmes Chinoises, Zhongguo xin nüjie $z a z h i$ 中國新女界雜誌, éditée la même année par Yan Bin 燕斌 (1869- ?) à Tokyo et qui tirait à 10000 exemplaires, Tianyi ne tirait qu'à 500 exemplaires (Lu $2015: 4)$. Mais il était considéré comme l'un des trois périodiques majeurs édités et animés par des femmes à Tokyo à cette époque, les autres étant le Journal des Femmes Chinoises, Zhongguo nübao 中國女報, de Qiu Jin, et celui de Yan Bin cité précédemment (Lu 2015 : 2).

Il y avait sûrement plusieurs raisons à ce tirage limité. D'abord, le contenu était rédigé en langue classique (par opposition à la langue vernaculaire, plus proche de la langue parlée), et les articles étaient moins ancrés dans des préoccupations «quotidiennes " (comme l'étaient ceux de la revue de Yan Bin, qui proposait davantage de vulgarisation scientifique, des conseils en tous genres ou des romans en épisodes...) ; il s'agissait en effet surtout d'essais théoriques. Cela constituait deux obstacles à sa large diffusion, dans le contexte d'un très faible taux d'alphabétisme et de scolarisation des femmes. 
9 Ensuite, ce journal portait des idées très « radicales » qui, de la part de femmes, les rendaient encore plus inaudibles. En effet, d'autres périodiques anarchistes, édités par des hommes, avaient une bien plus grande circulation et surtout duraient plus longtemps (à titre de comparaison, le journal Nouveau Siècle, Xin shiji 新世紀, fondé par quatre hommes en juin 1907, dont les contributeurs ne sont que des hommes, et qui aborde très peu les questions relatives aux droits des femmes, a « tenu » 121 numéros). Le constat est encore plus amer lorsque l'on regarde les dix-huit numéros, à large circulation, du Monde des Femmes, Nüzi shijie 女子世界, publié de 1904 à 1907 par une écrasante majorité d'hommes - sans que leur identité soit claire, soit parce qu'ils utilisent des noms qui ne sont pas genrés, soit parce qu'ils choisissent délibérément des pseudonymes de femmes (de type « Madame... ») pour écrire.

10 En comparaison, la revue de Yan Bin a dû interrompre la publication après seulement six numéros, pour des raisons principalement financières, comme l'indiquent les nombreux appels à dons et à financements dans la revue; les contributrices avaient pourtant même fondé leur propre imprimerie pour économiser les coûts d'impression et avaient annoncé un calendrier de publication allant jusqu'au onzième numéro. Aujourd'hui, même le dernier numéro, le numéro 6, a été perdu (Wang 2019). On peut aussi prendre l'exemple de tous les autres journaux de cette époque qui ont été fondés et animés par des femmes, et qui dépassent rarement dix numéros.

11 Comme d'autres femmes qui, à cette époque, fondent des associations et des dizaines de revues et de journaux, He-Yin Zhen a donc écrit et publié (bien qu'elle n'ait pas été retenue par l'historiographie). Pour le dire sommairement, elle a pu le faire parce qu'elle appartenait à une certaine élite, avait eu accès à l'éducation et à des moyens financiers suffisants (c'était là des conditions nécessaires étant donné la configuration de l'édition); mais elle a rencontré de sérieuses limites (pour s'en convaincre il suffirait de comparer le nombre d'ouvrages et d'essais publiés par Liu Shipei, et le fait qu'elle n'a pour sa part publié qu'une quinzaine d'articles), parce que c'était une femme.

12 Dans le sillage du Tianyi, Liu Shipei, empruntant apparemment le nom de He-Yin Zhen (Wan \& Liu 2010 : 4), fonde en avril 1908 le journal L'Équité (Hengbao 衡報), dont il devient l'éditeur et qui est composé de dix numéros, beaucoup plus courts. Il semblerait que, suite à la répression des autorités, iels quittèrent le Japon en novembre de la même année (Wan \& Liu 2010: 4), et si l'on a des indications biographiques sur Liu Shipei relativement fournies, on en a très peu sur He-Yin Zhen. Ce n'est pas un cas isolé, puisque pour la plupart des auteures de cette époque, on ne connaît que rarement leurs dates de naissance et de décès, et on ne sait presque rien sur elles, la seule source d'information étant les lettres ou hommages qu'elles publiaient les unes sur les autres dans leurs journaux, ce qui constitue une base alternative intéressante, mais insuffisante, d'informations.

13 He-Yin Zhen a commencé à attirer l'attention des chercheur.se's dans les années 1990, notamment par le nouvel intérêt porté à l'histoire intellectuelle de la fin de la dynastie des Qing et à l'anarchisme de la première moitié du vingtième siècle (Zarrow 1988, 1990). Ce n'est que dans les années 2010 cependant que He-Yin Zhen commence à être relue et étudiée dans le cadre de l'histoire du féminisme et des études de genre, que des analyses plus poussées sont proposées (Xia 2013,2014), et que ses idées sont discutées dans des mémoires et articles de recherche en Chine et à Taïwan. Ces études ont notamment été permises par la réédition complète des articles du Tianyi et du Hengbao par Lydia Liu et Wan Shiguo à Pékin en 2010, qui est accompagnée de nombreuses 
notes, et surtout permet d'avoir un accès facile aux textes (en dehors des archives ou des anthologies uniquement consultables dans des bibliothèques spécialisées). Les textes sont aussi très lisibles, car entièrement retapés, contrairement aux versions d'origine dont la lecture peut parfois s'avérer compliquée. On peut y retrouver une quinzaine de textes de He-Yin $\mathrm{Zhen}^{1}$, dont on a dressé une liste chronologique en fin d'article.

Cette réédition a donné lieu à une traduction de six de ces textes en anglais, version éditée par Lydia Liu, Rebecca Karl et Dorothy Ko en 2013, sur laquelle on aura l'occasion de revenir. Enfin, une traduction française a été proposée de ces six mêmes textes en $2018^{2}$.

En portant attention aux manières dont He-Yin Zhen développe quelques-unes de ses idées et en examinant le processus de traduction, on se posera les questions suivantes : Comment l'étude de son lexique permet-elle de percevoir l'hybridité des influences, et le rôle des traductions dans la formulation de sa pensée anarchiste et féministe? Comment cette hybridité permet-elle de rendre compte de l'impasse des dichotomies interprétatives "tradition - modernité ", «réforme - révolution» ou "Occident Chine » de ces textes, et plus généralement du début du vingtième siècle chinois?

Ensuite, comment He-Yin Zhen a-t-elle été (re) découverte, et donc reconstruite ? Dans quel lexique puise-t-elle pour exprimer ses idées ? Comment peut-on rendre ce lexique en français?

Enfin, comment la «libération des femmes» (nüzi jiefang 女子解放) peut-elle être envisagée comme un outil critique et épistémologique, qui nous amène aussi à nous saisir de la perspective féministe pour analyser et critiquer la production du savoir?

\section{Hybridité des influences}

\section{Féminisme anarchiste : la dialectique nan-nü}

He-Yin Zhen inscrit la «condition des femmes chinoises" dans l'historique et les mécanismes d'un rapport de force. En parlant d'oppression et de domination dans ses textes, elle se distingue nettement des discours des réformateurs de cette époque qui déplorent l'«obscurité » ou la "dépendance » des femmes, ces "Autres » décrites comme des êtres souffrants ${ }^{3}$.

En analysant les mécanismes de domination des «hommes»(nanzi 男子) sur les «femmes» (nüzi 女子), elle introduit une force politique et critique dans ses textes: jusque là en effet, les « hommes » était le nom du pouvoir relativement invisible à luimême, dont l'autorité avait effacé l'existence pour en faire l'universel, c'est-à-dire ce qui ne posait pas question. Les « hommes » n'apparaissent pas comme un acteur majeur du «problème des femmes », ni dans les textes de Liang Qichao 梁啓超 (1873-1929), ni dans ceux de Jin Tianhe (Jin Yi) 金天融 (1874-1947), deux intellectuels réformateurs de son époque qui ont écrit sur les «droits des femmes» (nüquan 女權). La domination masculine, parce qu'elle n'a pour eux, pas grand-chose à voir avec la «condition des femmes ", ne dit pas son nom. De manière intéressante, on peut alors remarquer que, dans les textes de He-Yin Zhen comme dans ceux d'autres auteures, se distinguent deux entités grammaticales genrées, d'un côté "nous, les femmes », et de l'autre "eux, les hommes » (Qin $2014: 6$ ). 
Une articulation importante de ses textes est l'expression nan-nü 男女, à savoir " homme-femme », ou "masculin-féminin », ou " entre les hommes et les femmes", dans le sens précis de la création et du renforcement historique et social d'une distinction ${ }^{4}$. Par la séparation genrée de l'intérieur (nei 内) et de l'extérieur (wai 外), et les interdictions auxquelles les femmes sont assujetties, celles-ci sont sommées de «s'occuper du foyer », « élever des enfants» et «servir leur mari ». Les femmes sont donc «utilisées» (liyong 利用) par les hommes. Elle écrit dans «La question de la libération des femmes »:

Le système (zhidu 制度) qui prévaut en Chine depuis des milliers d'années a réduit les femmes en esclavage, en les forçant à se soumettre et à obéir. Dans les temps anciens, les hommes faisaient en sorte que les femmes leur appartiennent (si nüzi wei jiyou 私女子為已有) afin d'empêcher qu'elles ne s'adonnent à la débauche (yin 滛). Pour cela, ils ont établi des enseignements politiques et rituels (zhengjiao 政教), dont l'objectif était la séparation (fang 防) des hommes et des femmes, et leur différenciation (bie 別); ceux-ci sont ainsi devenus des principes majeurs de l'univers (TY: 133).

19 Nan-nü est une expression relativement courante à cette époque; il semblerait que cette expression ait été mobilisée pour la traduction de "sexe» de l'anglais au mandarin, notamment dans les traductions des textes d'Herbert Spencer et Thomas Kirkup par Ma Junwu 馬君武 en 1902 (Liu 2000 a : 108 et Liu 2017 : 5). Le terme nan-nü était déjà chargé de sens et de références que le terme « sexe » n'avait pas en anglais : premièrement, le terme chinois présupposait une binarité relationnelle que le terme « sexe » n'avait pas, et deuxièmement ne faisait pas référence à des réalités biologiques mais à une articulation sociale.

He-Yin Zhen écrit dans le "Manifeste des Femmes » : «ce que l'on appelle homme(s) (nanxing 男性) ou femme(s) (nüxing 女性), a été fait ainsi par les coutumes sociales et par l'éducation » (TY : 43). Autrement dit, la différence a été construite, et elle n'est pas naturelle. C'est l'égalité qui est naturelle; en ce sens, cette égalité radicale était une égalité sans genre. C'est une conception légèrement différente de celle des «droits naturels » que développent d'autres auteur-e's à cette époque (He-Yin Zhen ne reprend que très peu le terme de "droits », on verra pourquoi).

He-Yin Zhen se saisit alors de cet outil pour y inscrire la dialectique marxiste. Dans le "Manifeste des femmes", elle montre que les coutumes ont fait des hommes les «maitres» (zhu 主) et des femmes les «esclaves» ( $n u$ 奴), et que les hommes sont considérés comme des «êtres humains" (ren 人) alors que les femmes sont considérées comme des «biens» ou de la "propriété » (caichan 財産), c'est-à-dire comme des «choses» (wu 物) (TY: 41). Ainsi, les femmes ne sont pas, en réalité, considérées comme des êtres humains (ou, membres de l'humanité, renlei 人類).

Le terme de «société » shehui 社會 est défini ainsi :

Notre société d'aujourd'hui est une société de classes (jieji shehui 階級社會), et la société de classes est la société des puissants (qiangzhe 强者). [...] Si l'on ne détruit pas la société, on ne pourra pas éliminer le pouvoir (qiangquan 强權), ni rétablir l'égalité universelle (renlei pingdeng 人類平等) (TY : 550).

La société qui est actuellement en place dans le monde appartient au système de classes (jieji zhidu 階級制度), né de la combinaison d'un grand nombre de pratiques injustes et inéquitables (He-Yin a : 137).

23 La société est dès lors le nom donné à une forme d'organisation sociale particulière, celle du «système de classes ». Le terme de «classes » (jieji 階級) est employé dans 
deux contextes : les classes 《riches》 et 《pauvres»(pin-fu 貧富) et les classes 《de sexe»(nan-nü 男女); elle montre que ces deux types de classes sont imbriqués :

Ceux qui, récemment, parlent de la révolution en des termes simplement économiques ignorent que les classes qui sont les plus nettement marquées dans le monde sont les classes nan-nü (nannü jieji 男女階級). [...] Vouloir abolir la société de classes, c'est d'abord commencer par abolir les classes nan-nü (nannü jieji 男女階級). Cela voudrait dire que, que l'on soit une fille ou un garçon, on puisse être élevé de la même manière, être éduqué de manière identique, avoir accès à des droits égaux, ainsi que les femmes ne soient pas soumises aux hommes et que les hommes ne puissent dominer les femmes, en bref que la manière dont les hommes traitent les femmes soit identique à la manière dont les femmes traitent les hommes (Annonce : 137).

En l'état actuel de mes recherches, je n'ai pas trouvé d'autre mention chez les auteur·e's chinois'e's de cette époque (premières années du $\mathrm{xx}^{\mathrm{e}}$ siècle) des «classes nan-nü » qui, comme on le voit dans l'extrait précédent, s'avèrent être un outil théorique extrêmement frappant. Nan-nü, une fois articulé à l'idée de classes, prend un sens dialectique et radicalement politique. En effet, elle ne fait pas de nan-nü des «catégories sociales d'identification du sujet » mais des positions dans un système de rapports de force (Liu 2017 : 10). Il contient en germe toute la théorie de He-Yin Zhen sur la lutte des femmes et les rapports de pouvoir. S'il faut, dit-elle, «supprimer » ou " tuer » les hommes, ce n'est pas, semble-t-il, à prendre au sens propre, mais au sens de l'abolition de la catégorie nan dans le rapport de force, et donc de la distinction même nan-nü qui induit un rapport entre dominants et dominées.

Selon la chercheuse Liu Jen-Peng (2017), les travaux comme ceux de Xia Xiaohong ou Lydia Liu ont souvent insisté sur la rupture entre He-Yin Zhen et les idées anarchocommunistes de son époque. Cependant, elle montre comment, si He-Yin Zhen mobilise des outils critiques d'un point de vue de la "libération des femmes", celle-ci s'inscrit, notamment par son lexique, dans la continuité de l'anarcho-communisme chinois d'une part, et dans le contexte de discussion sur les droits des femmes par les groupes socialistes à Tokyo d'autre part.

En effet, en Chine, les idées anarchistes s'étaient diffusées dès 1902 sous la forme de textes, de livrets et de groupes de discussion (Liu 2017 : 5). La pensée anarchiste ${ }^{5}$ avait été introduite dans un contexte de diffusion d'un vocabulaire et d'idées révolutionnaires antimandchous, qui insistaient sur la «violence » et la "revanche » contre la dynastie mandchoue des Qing - lexique dont on retrouve des traces évidentes dans les textes de He-Yin Zhen. Les termes de propriété collective (gongchan 共産), d'égalité (pingdeng 平等), de survie [liée aux inégalités économiques] (shengji 生計) et de privilèges (tequan 特權), qui forment des articulations majeures de son lexique, avaient été introduits et diffusés par les traductions de Bakounine en chinois, ce qui remet en question le caractère complètement «original » de ses outils présenté par exemple en introduction de la traduction anglaise (Liu, Karl \& Ko 2013 : 23). Selon Liu Jen-Peng, sa critique de la famille s'inspirait par exemple d'Engels, sa critique de l'État et de la société tirait des éléments de Bakounine, et la question centrale de la "propriété collective » était clairement inspirée de son traitement chez Kropotkine (Liu 2017 : 10).

Or, Tianyi proposait justement, dans chaque numéro, une rubrique spéciale pour les traductions, dans laquelle on trouve des textes historiques sur les anarchistes russes ou européen-ne's, des récits biographiques, des traductions d'essais (notamment la traduction, de l'introduction du Manifeste du Parti Communiste dans le numéro 15, mais 
aussi des traductions de Kropotkine et Tolstoï dans les numéros suivants), la plupart étant traduits à partir de l'anglais ou du japonais. Le journal Hengbao allait plus loin en proposant même quelques textes en anglais et en esperanto dans une rubrique dédiée.

D'autre part, à Tokyo, une approche socialiste de la libération des femmes avait été initiée dès 1904 par des hommes comme Katayama Sen 片山潜 (1859-1933), Toshihiko Sakai 堺利彦 (1871-1933), Kotoku Shusui 幸德秋水 (1897-1911) et des femmes comme Kanno Sugako 营野须贺 (1881-1911) et Kamigawa Matsuko 神川松子 (1886-1936) dont certaines des idées étaient diffusées par le biais du journal Heimin Shimbun 平民新聞 (Liu $2017: 8$ ).

Cependant, He-Yin Zhen émettait souvent des réserves, notamment par rapport à certaines de leurs revendications qui étaient communes à la plupart des organes socialistes, comme celle du droit de vote. Ses idées ne faisaient pas non plus forcément consensus au sein de milieux anarcho-communistes, puisque que le Hengbao publie par exemple en mai 1908 une lettre de Zhang Ji 張繼 (1882-1947) qui suggère que le vocabulaire de la "revanche des femmes » soit plutôt remplacé par des revendications comme celle de l'« amour libre», qui serait selon lui "plus adaptée» (HB : 758). Elle était donc à la fois relativement isolée, tout en s'insérant dans un réseau d'échanges, de débats et de certaines continuités (Liu $2017: 13$ ).

\section{Le « point de vue universel »}

Le concept de distinction et de hiérarchie chez He-Yin Zhen a pour fondement le renzhi 人治, ce que l'on peut traduire par « système de contrôle social » (créé par les humains, par opposition à «naturel»). L'expression renzhi renvoie au système de relations hiérarchiques qui organisent le corps social et moral dans la tradition confucéenne, en faisant référence aux "vertus et attaches", qui lient par exemple le sujet à son souverain, le fils à son père, ou encore l'épouse à son mari ${ }^{6}$. C'est, selon He-Yin Zhen, un obstacle à la libération des femmes. "Quand bien même les hommes et les femmes auraient accès à l'éducation, ils vivraient toujours dans un monde dominé par le système de contrôle social (renzhi)» (TY : 136). Ainsi, observe-t-elle, peu de femmes étudieraient la politique et la loi, et aucune d'entre elles n'entrerait dans l'armée ou la police. L'accès à l'éducation (qui était alors une des revendications principales des "droits des femmes») ne pouvait donc, en lui-même, constituer une avancée sans transformation plus profonde de la société.

31 Ensuite, la perspective de la «libération des femmes» (nüzi jiefang 女子解放) est mobilisée en tant qu'outil critique et épistémologique pour l'analyse et l'évaluation d'un corpus historique, linguistique et littéraire.

Dans son texte " Postface aux poèmes de Qiu Jin ", elle discute des talents littéraires et de l'œuvre de Qiu Jin (1875-1907). Elle écrit (TY : 560) : «Le système de contrôle social (renzhi) est l'instrument de destruction du talent (tiancai 天才, entendu comme talent naturel).» Ainsi le système de contrôle social «empêche (ou contient) le talent, en particulier pour les femmes ». "Qiu Jin a pu déployer son talent, parce qu'elle n'est pas restée enfermée par le système de contrôle social. » Les femmes ne peuvent produire ou créer sans l'abolition de ce système, qui est étroitement lié à la morale (confucéenne). Dans son texte "À propos des trois principes", elle explique que la morale est un " pouvoir déguisé » : 
Afin que l'épouse accepte de se plier à son mari, et afin qu'elle demeure chaste, l'obéissance au mari a été érigée en vertu (meide 美德) et le fait de quitter son mari a été nommé un grand vice ( $d a^{\prime} e$ 大惡). Ainsi la morale est entre les mains des puissants (qiangzhe 强者) et les puissants en font un outil pour se protéger (hushen 護身). [...] Le pouvoir contrôle les personnes (zhiren 制人) de manière visible, alors que la morale les contrôle de manière invisible (TY : 46). comme 《égalité » (pingdeng 平等), « équité », ou 《justice » (yi 義 ou gongli 公理) sont tous ancrés dans un imaginaire classique. Mais utilisés pour traduire ces idées socialistes ou anarchistes de l'anglais au mandarin, notamment par l'infatigable traducteur Ma Junwu, ils étaient réinvestis, fondus dans un nouvel idéal universaliste et moderne (Liu 2000 a : 106), qui fondait l'égalité dans un ordre naturel à la fois ancien (un temps d'avant l'apparition des "coutumes sociales ») et intemporel, mêlant des influences taoïstes, communistes, bouddhistes et chrétiennes ${ }^{8}$. Ces termes indiquaient un ancrage très marqué dans la culture lettrée, tout en s'insérant dans un nouveau cadre de pensée anarcho-communiste. La conséquence de cela est aussi la rencontre 
entre leur signification morale (ou éthique) et leur signification politique : en effet, l'équité ou justice $y i$ 義 sous-entendait que la distinction entre les riches et les pauvres, ou les hommes et les femmes, était inacceptable d'un point de vue moral, et rappelait aussi les principes de la tradition lettrée confucéenne (Liu $2017: 8$ ).

Tous ces éléments montrent alors qu'on ne peut élaborer une théorie de la traduction à partir du seul prisme «culturel» (sous-entendant que les termes appartiennent à une culture, et doivent être transposés dans une autre), car cette vue présume une culture unifiée, un corpus de références fermé, ou dichotomique (ici : Occident - Chine). On voit que le sens ne peut être déterminé culturellement. La traduction féministe doit interroger les idées de "culture» et de "nation». Afin d'embrasser toutes les pluralités des textes, et en se plongeant dans la traduction de termes qui étaient déjà hybrides et influencés par la traduction, on se retrouve face à face avec la richesse passionnante de l'histoire intellectuelle de cette époque. "Plutôt que de reconfirmer les frontières qui séparent les nations, les cultures, les langages et les subjectivités, la traduction montre qu'elles sont brouillées » (Simon $1996: 157)$.

Mais He-Yin Zhen ne porte pas seulement sa critique vers le "système social" confucéen. Elle définit la "[vraie] libération ", en opposition à plusieurs " conceptions étroites et idées fausses » de celle-ci. Parmi ces conceptions étroites (xiayi 俠意), elle identifie l'indépendance économique (en effet, dit-elle, faire entrer les femmes dans le prolétariat ouvrier ne va libérer personne), le droit de participation politique ou de vote (qui en réalité, donnent le pouvoir à une élite de femmes pour opprimer la majorité des femmes), la libération sexuelle (qui permettrait aux femmes de laisser libre cours à leurs " désirs charnels » et à la « débauche ", sans pour autant libérer leur esprit) et enfin le mariage monogame (qui, sous couvert d'une égalité numérique et d'une nouvelle morale, ne supprime pas pour autant l'oppression des femmes). Pour analyser ces " conceptions étroites », elle distingue d'une part, le nom ou le titre (ming 名) de la réalité (shi 實), en réinvestissant par là les réflexions, allant de Confucius (551-479 av. JC) aux mohistes ( $v^{e}$ siècle av. JC) sur le rapport ou l'opposition entre le «nom» et la "réalité ». Elle mobilise cette distinction pour montrer l'absence de correspondance entre le nom (ou l'idée) et la réalité, absence rendue encore plus évidente lorsqu'elle dit le «nom vide» (kongming 空名). Elle distingue d'autre part la libération physique (routi shang zhi jiefang 肉體之解放) de la libération de l'esprit (jingshen zhi jiefang 精神之解放).

Les femmes asiatiques, enthousiasmées par l'idée de civilisation à l'occidentale, pensent que les femmes européennes et américaines sont déjà parvenues à la libération, et jouissent pleinement du bonheur de la liberté et de l'égalité. Elles leur emboîtent le pas, et s'en contentent. Hélas ! Le moment de la révolution des femmes est venu, et je refuse que les femmes s'en tiennent seulement à obtenir ce genre de fausse liberté et fausse égalité ; j'espère au contraire qu'elles se battent pour en avoir de vraies (TY : 136).

La libération des femmes vise plutôt, selon elle, l'abolition du gouvernement et la mise en place de la propriété collective (gongchan 共產). He-Yin Zhen est influencée par les textes de Kropotkine dans son approche d'un mode d'organisation sociale basé sur le principe de la propriété collective et de l'idée d'entraide (TY : 167-169). Elle explique enfin que la « libération » ne peut être que le fait des femmes elles-mêmes, c'est-à-dire une libération « active " (zhudong 主動) et non « passive» (beidong 被動). En effet :

L'extension des droits des femmes (nüquan zhi shen 女權之伸) doit provenir de la lutte des femmes (nüzi kangzheng 女子抗爭) et non être donnée (fuyu 付與) par les 
hommes. Si les responsabilités sont imposées par les hommes, alors nous perdrons notre liberté [auto-détermination, ziyou 自由]. Si nos droits nous sont donnés par les hommes, alors nous dépendrons toujours d'eux. Théoriquement, nous serions "libérées", mais en réalité les droits obtenus dans cette libération appartiendraient à d'autres, et les femmes seraient toujours utilisées par les hommes et resteraient leurs auxiliaires. C'est pourquoi je dis: si les femmes désirent accéder au bonheur de la libération, cela doit se faire par leur propre quête (nüzi zhi ziqiu 女子之自求) et elles ne doivent pas attendre que leur libération vienne des hommes (TY : 138). chinois ne peut faire l'économie d'une réflexion sur les pratiques de traduction (cette fois, vers le mandarin) qui ont alimenté la production intellectuelle de cette époque. Comme l'ont montré plusieurs historien'ne's, la fin de la dynastie des Qing (1890-1911) est caractérisée par une " appropriation active » du savoir occidental : cela passe par la traduction d'ouvrages, mais aussi par la mise en place de cercles d'études et d'écoles, et l'envoi d'élèves à l'étranger.

44 Comme l'a très bien montré Angel Pino (2013) dans le cas de l'intellectuel Ba Jin 巴金 (1904-2005), l'écriture et la traduction se mêlaient souvent intimement, se nourrissaient l'une de l'autre, jusqu'au point où le texte une fois traduit pouvait être 
considéré comme une œuvre à part entière (dans le cas de la traduction d'Alexandre Berkman par Ba Jin, montre-t-il, les exemples avaient été remplacés, des parties retirées, et d'autres ajoutées). Autre exemple, lorsque Xue Shaohui 薛紹徽 (1866-1911) traduit et compile les textes biographiques de «femmes occidentales » en 1906, elle réécrit en grande partie les histoires (Fong, Qian \& Zurndorfer 2004 : chapitres 3 et 4). La traduction repose aussi sur des changements linguistiques, des (ré-) appropriations terminologiques, des redéfinitions. Cela induit de revoir la traduction comme une création littéraire, linguistique et théorique, à part entière. Or, justement, « la théorie de traduction féministe a pour objectif d'identifier et de critiquer l'enchevêtrement des concepts qui relèguent à la fois les femmes et la traduction en bas de l'échelle sociale et littéraire » (Simon $1996: 1)$.

Pour donner un exemple précis de cela, on peut prendre l'exemple du mot «bourgeois » qui, dans l'Introduction au Manifeste du Parti Communiste, publié dans le numéro 15 de Tianyi, fut traduit en chinois par shenshi fa 紳士閥 (à savoir, plutôt «familles de notables»), lui-même dérivé du japonais. Cela traduisait la réappropriation des concepts, qui n'étaient pas des catégories abstraites, mais faisaient sens par rapport à l'organisation sociale en Chine; les "familles de notables » représentaient alors la figure la plus plausible d'antagonisme au "peuple », dans un contexte encore faiblement industrialisé (Liu 2017 : 9). Il en changeait aussi le sens : les "familles de notables " possédaient les terres, leur richesse provenait principalement de l'agriculture et du commerce ; selon cette traduction, le prisme de l'opposition entre les capitalistes (des villes) et les ouvriers chez Marx devenait donc celui de l'opposition entre les riches et les pauvres pinfu 貧富, dans un imaginaire plutôt rural (Liu 2017 : 9).

Ainsi He-Yin Zhen utilisait la redéfinition et la typologie pour réinvestir les termes et leur signification, pour les mobiliser en tant qu'outils critiques et théoriques. Les termes qu'elle utilise (révolution, droits, libération) ne sont pas seulement des néologismes, qui auraient été employés pour adapter ou transposer des termes issus de langues européennes (Angle 1998: 19). C'est le cas, par exemple, pour «droits des femmes " (nüquan) et plus généralement «droits » (quanli), dérivés du japonais ${ }^{10}$. Les personnes qui entreprennent la traduction (et donc, la re-traduction) doivent donc se plonger dans « l'histoire de la langue, l'histoire du moment, et l'histoire de la-langueen-traduction » (Spivak 1993: 186). Les traductions qui paraissent dans les revues de l'époque, et leurs références, n'étaient jamais là "telles quelles", mais déjà lues et revues. Les idées, en circulant par la traduction, étaient sans cesse transformées.

Les traductions des textes des "droits des femmes » à la fin des Qing étaient d'ailleurs entreprises depuis une position genrée (majoritairement des hommes), et dans un contexte impérialiste et nationaliste.

En effet, à la fin de la dynastie des Qing (1644-1911), après la défaite des guerres de l'Opium du milieu du XIX $x^{e}$ siècle, puis la défaite de la Chine face au Japon (1894-1895) et la course aux concessions des puissances occidentales (l'Allemagne, la Russie, la France et le Royaume-Uni se partagent des ports et sécurisent leur présence sur le territoire), des intellectuel-le's lancent en 1898 un mouvement nationaliste de réformes politiques et sociales, dans lequel la «question des femmes » (funü wenti 婦女問題) devient un sujet incontournable. La "question" des femmes (on pourrait aussi traduire par " problème ") dérivait de l'idée, notamment diffusée par l'Anglais Herbert Spencer, que le degré de civilisation d'une nation se mesurait à l'aune de la «condition» de ses femmes. De cette doctrine impérialiste venait l'idée que les «deux cent millions de 
femmes » chinoises étaient devenues un ensemble numérique qui posait " problème » à la nation, et qu'elles devaient évoluer, au sens propre. La " question des femmes » était donc avant tout un discours sur les femmes, définies par leurs carences communes, leurs incapacités, leur ignorance, leur arriération (Qin 2014: 5). Elles étaient tenues pour responsables du «retard» imaginé de la Chine. Cela a encouragé ces intellectuelle's à défendre les "droits des femmes ", parmi lesquels le droit au travail et le droit d'aller à l'école, l'arrêt des "pieds bandés ", la fin du concubinage ; bien sûr, tous ces droits étaient strictement définis dans les limites des genres : des contenus enseignés dans les écoles aux nouveaux rôles de l'épouse, il ne s'agissait pas, pour ces réformateurices nationalistes, de venir bousculer fondamentalement les normes. De même, comme on l'a dit, le rôle des « hommes » dans l'oppression des femmes était un impensé relativement flagrant.

Dans ce cadre, la traduction de textes associés à la «modernité occidentale » répondait à l'anxiété des intellectuels chinois (ici, majoritairement des hommes) de rattraper le « retard» (perçu comme tel) et satisfaisait leur « désir impérial », leur soif de pouvoir ${ }^{11}$. Ainsi Liang Qichao expliquait-il que la traduction était une étape nécessaire à la renaissance de la nation (Liu $2000 \mathrm{a}: 76$ ). Au lendemain des défaites du XIX siècle, il leur paraissait clair qu'il fallait apprendre des Japonais et des Occidentaux pour les contrer, et pouvoir " être à leur place ", c'est-à-dire pour dominer. L'idée de "régner par la connaissance » n'était pas nouvelle, mais cette idée a alors été reconfigurée, à l'aune du darwinisme social, en désir de compétition pour « faire prospérer le pays et fortifier la race », fuguo qiangzu 富國强種 (Liu 2000 a : 101).

50 Liu Jen-Peng prend l'exemple de la traduction du texte "Les droits des femmes» d'Herbert Spencer par Ma Junwu, en montrant comment celui-ci cherche à embellir plus encore le "modèle anglais » en gommant systématiquement les nuances que Spencer y apporte (Liu $2000 \mathrm{a}$ : 115-122). Dans l'ordre symbolique où Ma Junwu était pris, il traduisait les hommes occidentaux, en s'identifiant à eux (Liu $2000 \mathrm{a}: 120$ ). Il s'accrochait donc à la distinction entre le modèle occidental et la " déchéance » locale. Car si l'Occident n'était pas parfait, pourquoi aurait-il eu besoin de le traduire?

51 Or, si la littérature sur les femmes d'un Herbert Spencer ou d'un Stuart Mill était un boys' club, telle était aussi la traduction de cette littérature dans les cercles réformateurs. Les femmes chinoises étaient présentes en filigrane comme l'entité qui était visée, devait évoluer, en clair, devait être supprimée; et si les «femmes occidentales » étaient convoquées, c'était en tant qu'attributs des civilisations (entendre, des hommes civilisés), et en tant qu'elles étaient désirées ou sexualisées (Liu 2000b). L'obsession pour le choc Occident/Chine, dérivée des récits impérialistes, empêchait de concevoir un rapprochement transnational entre les femmes parce que les notions de race et de nation étaient écrasantes (Liu 2000 a : 110).

Ainsi la traduction de ces récits des «droits des femmes » était-elle travaillée par le désir de connaître, de posséder, pour dominer et concurrencer ${ }^{12}$.

Cela est moins vrai de la traduction et de l'interprétation de textes anarchistes ou révolutionnaires, parce que ceux-ci empêchaient en grande partie l'idéalisation de l'Occident : au contraire, ils constituaient des ressources pour la critique de la «fausse civilisation » (wei wenming 偽文明) qui regorgeait d'inégalités, et autant de preuves des pièges des rhétoriques modernistes (TY : 148-149). Ils permettaient de penser une solidarité transnationale et l'universalité de l'oppression des peuples. Il est à noter que la plupart des cercles communistes ou anarchistes (Liu 2000 a : 102) s'appuyaient sur un 
système passionnant de correspondances et d'envois de documents depuis l'étranger ${ }^{13}$. Pour donner des exemples de l'intensité des circulations et traductions, on peut par exemple voir que le Tianyi publiait des comptes-rendus de réunions et assemblées organisées en Europe à peine un mois après leur tenue (TY : 304-313) et de nombreux textes quelques mois après leur parution en Europe (Liu 2017: 12). Si le désir de possession pour la mise en concurrence des races y était relativement absent, la traduction était toujours entreprise en grande majorité par des hommes (on retrouvait d'ailleurs Ma Junwu), ce qui avait pour résultat que les textes traduits étaient aussi en grande majorité des textes d'hommes, qui, lorsqu'ils parlaient des femmes, le faisaient en leur absence.

\section{Instabilités lexicales et historicisation des concepts}

S'interroger sur la traduction de significations non figées est essentiel, comme dans le cas des termes comme "égalité », «femmes » ou "droits », parce que ces termes sont disputés et redéfinis. Cela permet aussi de prendre des distances avec divers mythes essentialisants de la langue chinoise (qui nourrissent les fantasmes d'un "ailleurs ») ou la formation artificielle de concepts « essentiellement » chinois $^{14}$, et de plutôt concevoir la langue comme un espace de rivalités et de réappropriations, ce qu'illustrent bien les productions théoriques comme celles de He-Yin Zhen, et des intellectuele-s de son époque, mais aussi les pratiques d'aujourd'hui, celles des internautes et des militant. e. s LGBTQ+, et celles des groupes féministes locaux qui, du moins jusqu'aux vagues de répression et d'arrestations de 2015 , ont tenté de promouvoir un vocable critique du travail de contrôle sémantique du Parti Communiste Chinois et de la Fédération des Femmes (organisme national lié au Parti).

\section{Déconstruction d'une catégorie homogène « femmes »}

He-Yin Zhen montre qu'à l'intérieur des classes nan-nü, il y a encore d'autres classes. Elle dresse de longues typologies, en s'efforçant d'analyser les défis et oppressions spécifiques (les ouvrières, les mères, les domestiques, les concubines, etc.). Elle part aussi d'exemples dans lesquels les femmes oppriment d'autres femmes: les "maitresses de maison» maltraitent leurs domestiques, leur infligeant même des châtiments corporels, et des petites filles sont vendues par des mères pauvres à des époux riches. Une fois arrivées dans le foyer du mari, elles sont maltraitées par la mère de celui-ci et par les autres femmes qui "défoulent leur colère » sur elles, jusqu'à les mener parfois au suicide (TY : 218-219). He-Yin Zhen conclut que les femmes pauvres ne sont pas uniquement opprimées par des hommes, mais aussi par des femmes. Elle écrit alors : «notre intention est non seulement de rejeter le pouvoir des hommes sur les femmes, mais aussi de se révolter contre le pouvoir que des femmes imposent à d'autres femmes » (TY : 218).

Dans "La question de la libération des femmes ", elle évoque par exemple les travers des revendications de participation politique au sein des mouvements pour les droits des femmes: si les femmes votent et se présentent aux élections, une minorité de femmes riches entrera dans les cercles de pouvoir et obtiendra le pouvoir. Même si des femmes entrent au Parti Socialiste, cela ne servira pas les intérêts de la majorité des femmes ouvrières. La participation aux politiques parlementaires ne permet donc pas 
d'instaurer l'égalité universelle, et n'est qu'un titre vide (kongming 空名). He-Yin Zhen vise les intérêts de « la majorité des femmes » (duoshu nüzi 多數女子) ou de " toutes les femmes» (nüzi quanti 女子全體). Comme elle le répète à plusieurs reprises, la révolution des femmes ou la révolution nan-nü doit être une révolution " complète ", qui entraîne des transformations en profondeur (genben gaige 根本之改革), en renversant les structures du pouvoir et en abolissant l'oppression d'une minorité sur une majorité. C'est pourquoi, dit-elle, la révolution nan-nü doit ê tre menée conjointement avec, ou doit initier, les révolutions « raciales, économiques, sociales et politiques $»^{15}$.

Traduire les textes d'auteures comme He-Yin Zhen permet de redécouvrir, dans une optique féministe, les nombreuses pratiques discursives, méthodes de résistance et "stratégies rhétoriques subversives" qui permettent de renégocier les structures de genre (Bo 2010 : 5). Envisager les pratiques textuelles féministes et les outils critiques chez He-Yin Zhen revient aussi à s'interroger sur l'histoire de ces termes, et ce qu'ils sont devenus. Il faudrait pouvoir relire He-Yin Zhen en se défaisant du travail linguistique et historique de transformation des significations, afin de tenter de relire ces termes dans ce qu'ils pouvaient signifier à cette époque, pour elle et ses lecteur-rice's. Or, « le langage joue un rôle crucial dans la formation et le maintien des anciens rapports de genre ; le langage est donc également important pour en forger de nouveaux " (Bo 2010: 8). Tout au long du $\mathrm{xx}^{\mathrm{e}}$ siècle, les auteures continuèrent à retravailler les significations et à lutter pour l'expression du genre. Il faudrait donc traduire en tentant de rendre ces stratégies textuelles et ces instabilités lexicales.

D'autre part, il faut prêter attention aux formes et risques d'aplatissement, de lissage ou d'homogénéisation de la traduction. Il faut par exemple tenter d'interroger la construction discursive et historique de la catégorie "femmes", surtout lorsque l'on retrouve, dans les textes de cette époque, une dizaine de manières de renvoyer aux «femmes », avec des nuances différentes. Par exemple, traduire les mots nüzi 女子, nü 女, funü 婦女, nü tongbao 女同胞, nüjie 女界, nüren 女人 invariablement par «femmes » en français, sans mention du terme chinois, tend à masquer la pluralité des termes, et masque le fait que l'expression «femmes » n'est pas unique et inébranlable, mais une construction historique et politique.

La chercheuse Tani Barlow a montré comment l'invention d'un terme générique "femmes » en langue chinoise avait constitué un événement historique et lexical au début $d u x^{e}$ siècle - avant, argue-t-elle, il n'y avait pas d'agent unique qui renvoyait aux «femmes" en général, mais différentes catégories et positions dans la sphère sociale, auxquelles s'appliquaient des protocoles rituels spécifiques : fille non mariée, épouse, mère (Barlow 2004 : 41). Les «femmes" étaient produites comme catégories discursives selon leur place dans le cadre de la famille (jia 家) et dans un contexte relationnel (par rapport à tel ou tel individu masculin dans l'espace social). À partir des années 1890, explique-t-elle, les « femmes » ont été (re) théorisées au sein de la nation (guojia 國家) et de la société (shehui 社會), et le processus de « genrisation » n'était plus multiple mais binaire, le fameux nan-nü. L'invention de la catégorie funü 婦女 au milieu du xIX siècle est alors un événement relativement singulier : dans le terme funü, la femme mariée ( $f u$ 婦) est associée à la femme non mariée (nü 女) pour former le signifiant pluriel « femmes ». Il est intéressant de noter que He-Yin Zhen n'emploie pas le terme funü, mais celui de nüzi, qui se défait des connotations classiques. 

et de la traduction du terme « féminisme ». Souvent, l'argument principal qui cherche à discréditer des corpus en dehors d'un champ unique du féminisme est de dire : ce n'est pas féministe car le mot n'y est pas. Les implications ethno-centrées (et par là, linguocentrées) de cette affirmation sont profondes. Mais à l'inverse, plaquer le terme « féminisme » à tort et à travers ne masque-t-il pas les significations non figées dont on vient de parler? traduire nüzi 女子 par « féministe » dans le titre « Nüzi xuanbu shu 女子宣布書》 (《The Feminist Manifesto »), mais on pourrait aussi bien traduire nüzi par "femmes» (« Manifeste des femmes »), comme elles l'ont fait dans la traduction des expressions et titres des autres articles, comme le titre «Jingji geming yu nüzi geming 經濟革命與女子 革命 » traduit par « Révolution économique et révolution des femmes ». Il nous semble que, bien que la traduction «féministe » puisse correspondre au sens, par exemple, de l'expression nüzi jiefang (libération des femmes) telle que définie par He-Yin Zhen, cela induit une extrapolation sur le fait qu'elle se serait déclarée explicitement "féministe » et aurait appelé ses textes "féministes ». On pourrait aussi avancer que la traduction systématique de nüzi par «femmes » n'est pas forcément non plus une bonne option, parce que cela met l'accent sur le nom d'un groupe social plutôt que sur le contenu et les idées, mais ce point de traduction permet de lancer la réflexion.

64 Le terme « libération » jiefang 解放 dans « libération des femmes » nüzi jiefang 女子解放 est important à observer. Harriet Evans (2003) explique que ce terme, que He-Yin Zhen préfère au terme "droits », a contribué, au début du xxe siècle, à faire des "femmes " un groupe « révolutionnaire » et actif dans sa libération (contrairement au jiefang plus 
tardif du Parti Communiste, qui a été redéfini dans un sens de libération passive, car entreprise par le Parti, sur les femmes). Or, jiefang, chez He-Yin Zhen, était au contraire associé à l'idée d'action collective ; comme on l'a vu, elle donnait un rôle d'avant-garde aux femmes dans la libération collective.

L'historienne Wang Zheng (1999) fait remonter aux années 1910-1920 les expressions comme nüzi zhuyi 女子主義 ou nüquan zhuyi 女權主義, toutes deux employées pour désigner «féminisme » (respectivement, "théorie des femmes » et "théorie des droits des femmes"; zhuyi renvoyant à "doctrine/théorie» ou «-isme »). Cependant, il semble intéressant de considérer aussi la "libération des femmes » (nüzi jiefang) et la "révolution des femmes" (nüzi geming) de He-Yin Zhen comme des expressions originales des "féminismes » en Chine, des expressions plurielles pour nommer les féminismes dans l'histoire. La période 1904-1911 est à cet égard importante, avec la production de nombreux textes qui remettent en question les distinctions sociales du genre (chez Yan Bin par exemple) et appellent à une révolution des femmes, parfois violente et armée (chez Qiu Jin).

Ces termes évoluent. En s'institutionnalisant et en rentrant dans le giron du Parti, certains de ces termes, comme jiefang, ont perdu une grande part de leur portée critique (ce qui est visible aussi dans l'expression nannu pingdeng 男女平等 qui s'est vidée de toute tension dialectique). Cela ne signifie pas pour autant que les militantes féministes en Chine ne poursuivent pas parallèlement le travail d'invention et de contournement de la langue ${ }^{16}$.

\section{La « libération des femmes », un outil critique de la production du savoir}

Comme on l'a vu, He-Yin Zhen remet en question les "modèles" occidentaux autoproclamés d'une part, et le nationalisme instrumental des élites masculines chinoises d'autre part. Elle montre que les distinctions de genre sont des construits sociaux et qu'il y a des rapports de domination croisés. L'examen de ces idées s'est fait en lien avec une réflexion sur la traduction et sur les intérêts et désirs de la traduction, à différents niveaux. J'aimerais maintenant aborder des questions plus larges qui se posent lorsque s'amorce le projet de traduction: dans quel contexte historiographique et épistémologique s'insère-t-il ?

\section{Se faire une place dans l'histoire ou la bousculer?}

Wang Zheng (1999) a montré comment les femmes chinoises ont longtemps été évacuées de l'histoire, jusqu'à être évincées même de l'histoire des "mouvements des droits des femmes ». En effet, observe-t-elle, ces mouvements sont souvent abordés sous l'angle de la "question des femmes", comme un objet de discours et de débats produits par des hommes, en mettant de côté le rôle des femmes comme protagonistes de leur propre libération (Wang 1999: 13). Ainsi, les deux intellectuels Liang Qichao 梁 啓超 (1873-1929), et Jin Tianhe 金天融 (1874-1947) sont souvent présentés comme des théoriciens majeurs, et pionniers, des "droits des femmes", alors que leurs discours s'inscrivaient dans la "problématisation " des femmes dans la "crise de la nation" (Qin 2014: 5), et sous couvert de "les émanciper» leur assignaient aussi des rôles précis : ceux de bonnes épouses ou de bonnes mères (liangqi xianmu 良妻賢母), capables 
d'assister les hommes dans l'effort national et d'élever de bons citoyens (des garçons). Selon Wang Zheng, non seulement l'historiographie s'est calquée sur cette perspective masculiniste, mais elle s'est aussi davantage concentrée sur le mouvement politique du 4 mai 1919, puis des groupes de femmes rattachés au Parti Communiste Chinois après sa création en 1921, ce qui, dans les deux cas, amenait à minimiser la portée politique et critique de leurs revendications, et à sous-entendre que les femmes trouvaient « dans les mouvements et organisations politiques, l'encadrement nécessaire à leur prise de conscience et leur action» (Wang 1999 : 14).

Une première étape de la «redécouverte des femmes » dans l'histoire du début du $\mathrm{xx}^{\mathrm{e}}$ siècle a été, selon la chercheuse Rachel Leow, guidée par une approche biographique. Mais cette approche était souvent individuelle et romancée (Leow 2012: 2). Cette méthode était en réalité ancienne, car les biographies de "femmes exceptionnelles » étaient déjà courantes à la fin des Qing. Mais on peut effectivement remarquer que «l'histoire des femmes chinoises » a grandement été prise, jusque dans les années 2000, dans une approche réservée aux « héroïnes-martyrs ». C'est le cas pour Qiu Jin 秋瑾, ou encore pour Xiang Jingyu 向警予, 1895-1928 (Hu 2007), des femmes «remarquables", dont le point commun était la ferveur nationaliste et révolutionnaire, et qui donc pouvaient facilement être digérées dans le récit national (Hu 2007 : 3). Surtout, le fait qu'elles soient présentées comme "exceptionnelles" emp êchait de bousculer réellement le récit historique qui les excluait d'emblée. Cela ne faisait que remplir un petit quota de femmes, dans la marge (Leow $2012: 2$ ). Elles n'y étaient présentes qu'en tant que femmes, et ne constituaient qu'une petite poignée de femmes; ainsi elles étaient toujours déjà mises en scène, mises en valeur, montrées comme telles, alors que les cadres se donnaient pour transparents. On peut se demander, pour reprendre l'idée de Gayatri Chakravorty Spivak (1988), si cela ne les empêche pas de "parler » tout court, produisant l'inverse de ce qui est annoncé. Leur « mise en scène » occultait aussi la masse silencieuse des femmes anonymes, des femmes qui n'étaient pas assez exceptionnelles pour rentrer dans l'histoire. On pourrait d'ailleurs faire ce genre de reproche aux méthodes biographiques de « l'histoire des femmes » en général.

Depuis les années 2000, un certain nombre de travaux ont permis d'interroger ces récits et de révéler la participation massive des femmes dans les mouvements politiques et sociaux au début du xxe siècle (Wang 1999), la production intellectuelle des femmes dans la presse et l'édition à cette même époque, ainsi que leur rôle dans les réformes de 1898 (Qian 2003).

71 Et s'il s'agissait là de faire du féminisme, à l'image de la « libération des femmes » de He-Yin Zhen, un outil critique pour interroger la (re) production du savoir ? Et si, pour reprendre les formulations de Monique Wittig (1992), le "point de vue " féministe (comme celui de He-Yin Zhen, et de ses pratiques de traduction) n'était pas un " autre » point de vue, un point de vue minoritaire ou concurrent que l'on découvrirait dans les " marges ", mais bien un point de vue universel ? Ce point de vue constituerait un défi à l'homogénéisation et aux tentations d'exclusion, aux tentations de définition d'une expérience indifférenciée et de fermeture d'un corpus unique. Ce point de vue interrogerait les catégories de production de connaissances, l'historiographie sélective, ainsi que les intérêts et les désirs qui travaillent la production intellectuelle et la traduction. 


\section{Le processus de « visibilisation » au risque de la reproduction}

s'éloigne des idées des réformateurices nationalistes sur les «droits des femmes », cela mène les lecteurices à penser à une sorte d'opposition théorique entre des hommes nationalistes et une femme anarchiste, qui gomme le fait qu'il y avait aussi beaucoup de femmes nationalistes (qui portaient aussi des discours masculinistes), et vice-versa, que des hommes comme Liu Shipei avaient aussi des visions proches de celles de He-Yin Zhen sur la libération des femmes. Cela entretient donc une « exceptionnalisation » de He-Yin Zhen, sans outil pour comprendre la continuité des liens et des influences.

Concernant la traduction française, vraisemblablement inspirée de la version anglaise du texte, l'édition a choisi de titrer le recueil La Revanche des femmes et autres textes. Ce titre peut poser question parce qu'il suggère un sensationnalisme assez loin des textes qui, comme on l'a vu, dépassent le cadre de la «revanche des femmes» (cette expression est d'ailleurs très peu employée par He-Yin Zhen elle-même, sinon dans le titre d'un de ses textes). 
77 Mais il semble que les deux choix de titre (celui de la version anglaise et celui de la version française) soient surtout des stratégies de promotion de l'ouvrage, ce qui, dans les deux cas, est assez efficace, et montre les stratégies différentes des deux éditions : dans le premier cas, un aspect plutôt théorique et historique (avec une couverture sobre et grise), dans le deuxième, plutôt un attrape-l'œil, avec une connotation provocatrice et révolutionnaire (ce que suggère aussi la couverture rouge et noire de l'ouvrage). Les titres et les couvertures ont aussi une fonction de représentation et de reconstruction de la figure de He-Yin Zhen et de ses textes.

De plus, comme pour toute "naissance " ou "éveil $»^{19}$ se pose la question d'une périodisation contestable. Qu'y avait-il avant? Qui décide de la date de naissance ? Les personnes impliquées dans cette naissance percevaient-elles cela ainsi? L'auraientelles nommée autrement? Quelles continuités la métaphore de la naissance dissimulet-elle?

Or, ce que la traduction ne mentionne pas, c'est qu'elle reprend en réalité une périodisation très claire: les textes de Liang Qichao et de Jin Tianhe datent respectivement de 1897 et 1903, alors que les textes de He-Yin Zhen datent de 1907. L'historienne Yun Zhu distingue justement entre la période 1898-1904, davantage axée sur l'idée des droits des femmes et profondément tributaire du cadre nationaliste, et la période 1905-1911, qui voit aboutir davantage de réflexions sur la construction sociale du genre et la critique du rôle des hommes dans l'oppression des femmes (Yun 2017 : 16). Cette périodisation permet aussi de repenser le cadre interprétatif «nationalistes progressistes (hommes) / anarchistes (femmes)» en replaçant les idées dans des temporalités précises.

80 Enfin, on peut s'interroger sur le choix de ces six textes. Si ces textes semblent indubitablement être fondamentaux pour comprendre la pensée théorique de He-Yin Zhen, on peut noter qu'ils appartiennent tous à un type particulier : ce sont des essais philosophiques et politiques. D'autres textes, qui peuvent paraitre "marginaux », permettent cependant de voir comment elle applique ses idées, et permettent de nuancer la distinction entre théorie et pratique: par exemple, ses lettres ou ses discours, mais aussi le texte qui constitue son association («Principes de l'Association pour la restauration des droits des femmes »), ou enfin les commentaires qu'elle fait d'autres œuvres ou d'autres textes (comme sa «Postface aux poèmes de Qiu Jin »). De fait, le choix qui est fait de ces six textes induit la reproduction d'un «canon» qui exclut d'autres types de textes.

81 Ces quelques remarques visent donc à révéler les choix de sélection et de (re) présentation qui ont été effectués dans la publication de ces traductions, dans un effort pour visibiliser l'acte de représentation et de production du savoir.

\section{Interroger les discours sur la Chine}

82 Comme nombre de traditions universitaires et institutions françaises (on prend ici l'exemple de la France, mais on pourrait bien sûr élargir la réflexion), la sinologie, aujourd'hui rebaptisée "études chinoises» au sein des "aires culturelles» des universités, est historiquement fondée sur la traduction et la mise en récit. Elle est initiée au XVI ${ }^{\mathrm{e}}$ siècle par des missionnaires catholiques et jésuites comme Matteo Ricci (1552-1610), et fondée sur des principes et des méthodes coloniales. 

projet. Comme l'a bien montré Tejaswini Niranjana dans Siting Translation, la production de savoir des colonisateurices sur les colonisée's s'est faite par la traduction, et une conception de la traduction qui « présupposait la transparence de la représentation » (Niranjana 1992 : 12). En prenant l'exemple de l'Inde colonisée, elle montre comment les «découvertes, collections et traductions » participaient de l'entreprise de «domestication de l'Orient » et sa transformation en une " province du savoir européen »; l'entreprise de traduction fournissait et décidait de la place des colonisé. e. s dans l'architecture du savoir et le récit téléologique de la "civilisation » (Niranjana 1992: 15). Ces remarques sont absolument nécessaires pour réfléchir aux implications de la traduction dans un contexte post- (ou néo-)colonial. La chercheuse Liu Jen-Peng s'est inspirée des travaux de Tejaswini Niranjana, et a montré comment, si la Chine n'a jamais été " colonisée » au sens propre, le rapport de force impérial ou colonial est lui bien ancré dans l'histoire de la Chine, notamment du point de vue de l'histoire intellectuelle (Liu 2000 a : 95-102).

Le projet de traduction est alors empêtré dans des formes de violence coloniale et patriarcale, qui garantissent aux corpus et langues dominantes un accès privilégié à l'universalité ; ce projet participe d'une forme d'assimilationnisme occidental. Il est nécessaire de remettre en question, toujours, les méthodes et les «manières » de la traduction, en critiquant ses assises sexistes - notamment les connotations des métaphores des théories de traduction, comme celle de la "fidélité " au texte (voir Simon 1996, chapitre 1) - et coloniales (dont on verra des exemples plus loin), et en mobilisant des outils de critique féministe du pouvoir et de la représentation. Ces outils permettent d'interroger les discours produits sur la « Chine».

\section{Interroger le récit ethnocentré de l'histoire du féminisme}

Il y a un premier risque de perception et de construction des "féminismes chinois » à l'aune des catégories analytiques occidentales. C'est ce qu'explique la chercheuse Bo Wang (2010) : certain'e's chercheureuses ont arrêté un cadre et une définition du "féminisme ", une périodisation historique, des outils d'analyse, un corpus et une origine établie, qui refusent d'être déstabilisés, tout cela étant bien sûr fortement ethnocentré. On peut cependant considérer que le féminisme est une pensée moderne sans origine unique, sans corpus unique, sans langue unique ${ }^{20}$. Il apparaît alors fondamental de corriger l'idée d'une histoire du féminisme en Chine qui ne serait que l'histoire de « réponses chinoises au féminisme occidental » (Nan \& Wang 2015 : 5). Il existe en effet une forme de « rhétorique amnésique » selon laquelle la Chine ne serait qu'une « nouvelle-venue », un réceptacle passif du féminisme.

La perspective historique nous amène à nous interroger sur la complexité de la circulation des idées, des stratégies textuelles et de l'hybridité des modèles et des 
influences, et ainsi à « éviter le piège d'une construction historiographique de la pensée féministe procédant d'une vision téléologique et linéaire » (Möser 2013:6).

On sera parfois surprise's de découvrir des alliances politiques imprévues. Ainsi le récit monolithique du «féminisme blanc» rencontre-t-il des récits nationalistes en Chine qui considèrent le féminisme comme une « invention occidentale dangereuse » qui irait à l'encontre de la "tradition nationale », ou bien, dans une autre version, qui diviserait le peuple par ses aspirations petites-bourgeoises et individualistes ${ }^{21}$. Ce que ces deux récits, le récit du féminisme blanc et le récit nationaliste chinois, ont en commun, c'est le refus de considérer le féminisme comme une pensée et un mouvement hybride et transnational de résistance à l'oppression; les conséquences pratiques, concrètes, de ces discours n'ont pas encore été assez étudiées.

Enfin, certains travaux présentent des tentatives de définitions d'un "féminisme à caractéristiques chinoises", un féminisme "essentiellement» chinois, par les fantasmes linguistiques (comme ceux sur une langue féminine ancestrelle, le nüshu 女 書), sur des restes de sociétés matrilinéaires où se pressent les anthropologues ${ }^{22}$, ou encore l'espace mythologique d'une alternative idéale à l'Occident (voir notamment l'ouvrage Des chinoises de Julia Kristeva [1974] ${ }^{23}$ ). Ces récits se basent sur l'essentialisation (à la fois des femmes et de la Chine) et sur la fabrication de mythes « originels ».

91 C'est alors qu'apparaît l'utilité épistémologique du féminisme comme outil critique qui implique «un mouvement métadiscursif sur la manière dont les pays et les peuples sont étudiés et projetés» (Nan \& Wang $2015: 12$ ). Ce mouvement est marqué par un scepticisme envers les « récits englobants », et une attention particulière est portée aux « routes multiples et détournées par lesquelles le savoir est produit » (Judge $2015: 3$ ).

Les récits englobants, qu'ils soient nationalistes, orientalistes ou issus de théories niant certaines idées à certaines langues ou peuples, reposent toujours sur l'opposition entre deux « aires culturelles » prétendument distinctes, l'opposition Occident-Chine, dont la chercheuse taïwanaise Liu Jen-Peng (2000a) retrace de manière passionnante les fondements coloniaux et impérialistes. Elle montre comment, à la fin de la dynastie des Qing (1890-1911), les productions intellectuelles et littéraires, mais aussi la traduction, qui abordent ce qui s'appelait alors la "question des femmes ", expriment les intérêts et désirs d'une classe d'hommes, appartenant aux élites intellectuelles urbaines. C'est avec ces éléments en tête que la traduction des textes de He-Yin Zhen offre un exemple de défi à ces récits hégémoniques.

Comme cet article l'a montré précédemment, traduire est loin d'être un acte anodin. Il faut donc travailler aux méthodes propres à la traduction féministe critique. Comme le dit en substance Gayatri Chakravorty Spivak (1993), plutôt que de concevoir la traduction dans "la transparence de la représentation", ou dans des images masculinistes, cela suppose de "se rendre » au texte, d'être déstabilisées et de perdre son pouvoir sur le texte. Il faut envisager une traduction qui reconnaisse clairement l'intervention de la personne qui traduit : "le féminisme permet à la traduction d'être vue comme une forme de réécriture dans un contexte historique, social et culturel spécifique, une réécriture qui implique la subjectivité du [de la] traducteur [rice] » (Yu 2015), et ainsi mettre fin à l'invisibilité de la traductrice et de sa position sociale et genrée; tout comme la sinologie n'a jamais été une science "sans point de vue », ou « sans Sujet ». 


\section{Remarques conclusives}

L'objectif de traduction des textes de He-Yin Zhen est de se frayer un passage entre la violence coloniale de la traduction d'une part, et son utilité politique pour les solidarités féministes d'autre part. Les traductrices et éditrices de la version anglaise des textes de He-Yin Zhen indiquent une " théorie transnationale ». Mais comment (re) construire une «théorie transnationale " ? Comment, aussi, rendre audibles les idées $\mathrm{du}$ texte sans surcharger la lecture et donner un sentiment d'étrangeté ou d'intraduisible (et glisser sur des pentes orientalisantes et réifiantes), ou au contraire, surdéterminer le sens (en plaquant des interprétations) ?

Cette idée d'une théorie transnationale, aussi délicate soit-elle, est utile pour penser l'histoire textuelle et intellectuelle du féminisme. En effet, le féminisme a sans cesse été nourri par les traductions et les circulations. Sans ignorer les rapports de force coloniaux et les tentations nationalistes ou essentialistes, la traduction féministe propose quelque chose d'une "culture transnationale» dont parlait Homi Bhabha (1994: 212) : quelque chose qui ne peut se défaire des tensions de l'hybridité, et qui remet en question la sécurité de la «fermeture " (Simon 1996 : 128), en appelant à de nouvelles formes d'identification, de construction des subjectivités, de formulation des expériences, et d'analyse des systèmes oppressifs.

Traduire des textes de langue chinoise pour "élargir le corpus ", sans pour autant remettre en question les fondements occidentalocentrés des études féministes et études de genre, ne revient-il pas, finalement, à remplir ce fameux "quota d'inclusivité ", ajouter un chapitre sans changer la structure du livre, sans s'interroger sur les fondements coloniaux et racistes des disciplines universitaires comme la sinologie? S'interroger sur ces fondements serait, au contraire, initier ce que He-Yin Zhen appelle une "transformation en profondeur», par opposition à des « changements en surface » ou des propositions séduisantes mais tout à fait « vides ».

Ces questionnements rendent indispensable d'accompagner les pratiques de traduction de revendications politiques et féministes, et de militer pour faire sortir ces traductions des perspectives dites «d'aires culturelles (ou linguistiques)». Ces réflexions débouchent aussi sur les possibilités d'organiser des pratiques de traductions collaboratives, sous forme d'ateliers et de plateformes. S'obliger à se plonger dans l'histoire des textes et de la langue permet d'écarter les paradigmes d'interprétation trop simples pour s'interroger sur l'origine hybride des mots et les enjeux de leurs emplois et leurs transformations de sens. Cela implique aussi des pratiques interdisciplinaires et translinguistiques qui interrogent les récits de l'altérité irréductible et les mythes essentialisants.

Bien sûr, la traduction n'est pas une entité séparée : ces questionnements appellent à des transformations dans l'édition, l'université, et aussi dans les sociétés et les cadres de réception des traduction. À cet égard, il semble nécessaire de lutter pour l'ouverture de l'université, le renouvellement des méthodes et des découpages, la mise en accès libre et gratuite des traductions et des travaux, et l'organisation de moments d'échange et de réflexions sur la traduction et sur la production du savoir, avec une critique de leurs héritages problématiques.

Cela étant dit, la traduction est un moment capital de la circulation des idées politiques, du "voyage des théories", de leurs transformations, et de la construction des 
solidarités féministes. C'est pourquoi il faudrait sans cesse recommencer la réflexion sur la traduction, sans pour autant y renoncer.

\section{BIBLIOGRAPHIE}

ALI, Zahra. 2012. Féminismes islamiques, Paris : La Fabrique.

ANGLE, Stephen. 1998. « Did someone say rights ? Liu Shipei's Concept of Quanli » Philosophy East and West $48(4): 623-651$.

BARLOW, Tani. 2004. The Question of Women in Chinese Feminism. Durham : Duke University Press, Next Wave : New Directions in Women's Studies.

BHABHA, Homi. 1994. The Location of Culture. Londres; New York : Routledge.

BO, Wang. 2010. « Engaging Nüquanzhuyi: The Making of a Chinese Feminist Rhetoric » College English 72(4) : 385-405.

EVANS, Harriet. 2003. " The language of liberation: gender and jiefang in early Chinese Communist Party discourse ", in Twentieth-century China: new approaches, WASSERSTROM, Jeffrey (éd.). Londres ; New York : Routledge : 193-220.

FONG Grace, QIAN Nanxiu \& ZURNDOFER Harriet. 2004. Beyond Tradition and Modernity: Gender, Genre and Cosmopolitanism in Late Qing China. Leiden : Brill.

HONG FINCHER, Leta. 2018. Betraying Big Brother : the Feminist Awakening in China. New York: Verso Éditions.

HU, Ying. 2007. « Qiu Jin's Nine Burials: The Making of Historical Monuments and Public Memory » Modern Chinese Literature and Culture 19(1) : 138-191.

JUDGE, Joan. 2015. « Sinology, Feminist History, and Everydayness in the Early Republican Periodical Press » Signs 40(3) : 563-587.

KRISTEVA, Julia. [1974] 2001. Des chinoises. Paris : Pauvert.

LEOW, Rachel. 2012. « Age as a Category of Gender Analysis: Servant Girls, Modern Girls, and Gender in Southeast Asia » The Journal of Asian Studies 71(4) : 975-990.

LI, Youning \& ZHANG, Yufa. 1975. Jindai zhongguo nu quan yundong shiliao, 1842-1911 [Documents sur l'histoire des mouvements pour les droits des femmes chinoises de l'époque moderne]. Taiwan : Guoli taiwan daxue, lishi yanjiu suo, Zhuanji wenxue she.

LIU, Jen-Peng. 2000a. " Zhongguo de nüquan, fanyi de yuwang yu Ma Junwu Nüquan shuo yijie », in Jindai zhongguo nüquan lunshu : guozu, fanyi yu xingbie zhengzhi, LIU Jen-Peng éd. [« Le désir de traduire et le désir en traduction : le féminisme "chinois" et la traduction de Ma Junwu des Droits des Femmes » dans Récits des droits des femmes de la Chine moderne: politiques de la nation, de la traduction et du genre]. Taipei : Taiwan xuesheng shuju, 75-128.

LIU, Jen-Peng. 2000b. « Xifang meiren yuwang li de zhongguo yu er wanwan nüzi : wenqing yiqi wusi de guozu yu funü », in Jindai zhongguo nüquan lunshu : guozu, fanyi yu xingbie zhengzhi, LIU JenPeng éd. [" Désirs et fantasmes des "belles femmes occidentales”, la Chine et les femmes », in 
Récits des droits des femmes de la Chine moderne : politiques de la nation, de la traduction et du genre].

Taipei : Taiwan xuesheng shuju, 129-200.

LIU, Jen-Peng. 2017. « Tianyi de wuzhengfu gongchan zhuyi shiye yu He Zhen de nüzi jiefang » [ La théorie anarcho-communiste du journal de la Justice Naturelle et la "libération des femmes" selon He Zhen »], Funü yanjiu luncong 140.

LIU Lydia, KARL Rebecca, \& KO Dorothy. 2013. The Birth of Chinese Feminism: essential texts in transnational theory. New York : Columbia University Press.

LIU, Peizhu. 2020. «Countryside Feminism on Weibo: a citizen sociolinguistics perspective », Citizen Sociolinguistics, consulté en ligne le 20 février 2020. URL : https://

citizensociolinguistics.com/2020/01/01/田园女权-countryside-feminism-on-weibo-a-citizensociolinguistics-perspective/

LIU, Ting. 2008. « Cyberactivism in the Women's Movement: A Comparison of Feminist Practices by Women Organizing in Mainland China and Hong-Kong ", dans Chinese Women and the Cyberspace, KUAH-PEARCE Khun Eng (éd.). Amsterdam : Amsterdam University : 95-116.

LU, Chun-Hsien. 2015. «Wanqing Zhongguo xinnüjie yu tianyi bao nüxing lunshu bijiao chutan » [ Comparaisons entre la revue des Nouvelles Femmes Chinoises et le journal Justice Naturelle »] Wenxue qianzhan $15: 1-20$.

MÖSER, Cornelia. 2013. Féminismes en traductions. Théories voyageuses et traductions culturelles. Paris : Éditions des Archives Contemporaines.

NAN Z. Da \& WANG Zheng. 2015. «Feminist Sinologies : An Introduction » Signs 40(3) : 545-562.

NIRANJANA, Tejaswini. 1992. Siting Translation: History, Post-Structuralism, and the Colonial Context. Oakland : University of California Press.

PINO, Angel. 2013. « Ba Jin et Berkman, de la traduction à l'écriture palimpseste » À contretemps 45, consulté le 4 novembre 2019. URL : http://acontretemps.org/spip.php?article455

QIN, Fang. 2014. « Xin huici, xin shijie : qingmo minchu 'nüjie' yici tanxi » [« Nouveau mot, nouveau monde? Analyse du terme nüjie à la fin des Qing et au début la République »] Qingshi yanjiu 4.

QIAN Nanxiu. 2003. « Revitalizing the Xianyuan (Worthy Ladies) Tradition: Women in the 1898 Reforms » Modern China 29(4) : 399-454.

SIMON, Sherry. 1996. Gender in Translation: Cultural Identity and the Politics of Transmission. New York : Routledge.

SPIVAK, Gayatri Chakravorty. 1988. "Can the Subaltern Speak?», in Marxism and the Interpretation of Culture, NELSON Cary \& GROSSBERG Larry (éd.). Londres : Macmillan Education : 271-313.

SPIVAK, Gayatri Chakravorty. 1993. " The Politics of Translation ", in Outside in the Teaching Machine, New York : Routledge, 179-200.

SUN, Yifeng. 2018. Translating Foreign Otherness : Cross-cultural Anxiety in Modern China. New York : Routldege.

TY : WAN Shiguo \& LIU Lydia. 2010. Tianyi, Hengbao [Réimpressions du Tianyi et du Hengbao]. Pékin : Zhongguo renmin daxue chubanshe, Qingshi yanjiu congshu.

WAN Shiguo. 2011. «He Zhen nianbiao » [ Biographie chronologique de He Zhen »], dans Yangzhou wenhua yanjiu luncong, Yangzhou : Guangling shushe, 80-101. 
WAN Shiguo \& LIU Lydia. 2010. «Introduction », dans Tianyi, Hengbao [Réimpressions du Tianyi et du Hengbao]. Pékin : Zhongguo renmin daxue chubanshe, Qingshi yanjiu congshu, 1-27.

WANG, Qingyi. 2019. «Xin faxian de zhongguo xin nüjie zazhi diliu qi ji qi kaocha » [« Découverte du sixième numéro de la revue des Nouvelles Femmes Chinoises et analyse »], Chuanmei guancha 421.

WANG, Zheng. 1999. Women in the Chinese Enlightenment: Oral and Textual Histories. Oakland : University of California Press.

WITTIG, Monique. [1992] 2001. « Le Point de vue, universel ou particulier », in La Pensée Straight. Editions Balland. 51-54.

WU, Angela Xiao \& DONG Yue. 2019. « What is made-in-China feminism(s) ? Gender discontent and class friction in post-socialist China »Critical Asian Studies 51(4).

XIA, Xiaohong. 2013. Wanqing baokan, xingbie yu wenhua zhuanxing [Les journaux de la fin des Qing, le genre et les transformations culturelles, ouvrage]. Pékin : Renjian Chubanshe.

XIA, Xiaohong. 2014. « Wanqing nübao zhong de guozu lunshu yu nüxing yishi : 1907 nian de duoyuan chengxian » [ « Les récits nationaux et la conscience féminine dans les journaux de femmes de la fin des Qing : le foisonnement de l'année 1907 »]. Beijing daxue xuebao : 12.

XIONG, Yuezhi. 2008. « The Theory and Practice of Women's Rights in Late-Qing Shanghai, 1843-1911 », éd. et trad. Michael Ouyang, in Beyond the May Fourth Paradigm: In Search of Chinese Modernity, CHOW, Kai-wing, HON, Tze-ki, IP, Hung-yok \& PRICE, Don C. (éds.). Lanham ; Boulder ; New York ; London : Lexington Books : 71-92

YU, Zhongli. 2015. Translating Feminism in China: Gender, Sexuality and Censorship. Londres ; New York : Routledge.

YUN, Zhu. 2017. Imagining Sisterhood in Modern Chinese Texts 1890-1937. Lanham ; Boulder ; New York ; London : Lexington Books.

ZARROW, Peter. 1988. « He Zhen and Anarcho-Feminism in China » The Journal of Asian Studies, 47(4) : 796-813.

ZARROW, Peter. 1990. Anarchism and Chinese Political Culture. New York : Columbia University Press.

\section{ANNEXES}

\section{Sources : textes de He-Yin Zhen}

Ces textes ont été consultés dans la réédition suivante, et leur numérotation de pages suivent donc la pagination de cet ouvrage : WAN Shiguo \& LIU Lydia. 2010. Tianyi, Hengbao [Réimpressions du Tianyi et du Hengbao]. Pékin : Zhongguo renmin daxue chubanshe, Qingshi yanjiu congshu.

Les références de pages dans l'article sont précédées de TY lorsque les textes se trouvent dans le journal Tianyi et de HB lorsque les textes se trouvent dans le journal Hengbao.

《Légende au portrait de la déesse Nüwa » 女媧像並贊 Nüwa xiang bing zan 10 juin 1907, Tianyi $1: 3$.

«Manifeste des femmes »女子宣布書 Nüzi xuanbu shu, 10 juin 1907, Tianyi 1 : 41-44. 
«À propos de trois principes [sous-parties : les monarques et les prostituées, les voleurs et les gouvernements, la morale et le pouvoir] » 公論三則 [帝王與娼妓，大盜與政府， 道德與權力], Gonglun sanze [diwang yu changji, dadao yu zhengfu, daode yu quanli], 10 juin 1907, Tianyi $1: 46-49$.

《Lettre de Zhen aux étudiantes chinoises au Japon »震致留日女學生書 Zhen zhi liuri nüxuesheng shu, 10 juin 1907, Tianyi 1 : 547-548.

《Introduction aux peintures de Su Manshu » 曼殊畫譜序 Manshu huapu xu, 10 juin 1907, Tianyi $1:$ 551-554.

《Principes de l'Association pour la restauration des droits des femmes » 女子復權會簡 章Nüzi fuquan hui jianzhang, 10 juin 1907, Tianyi 1 : 581-583 [co-écrit par He-Yin Zhen, Lu Huiquan, Zhang Xu, Zhou Dahong, Tong Qi et Xu Yazun].

«À propos de la revanche des femmes » 女子復仇論 Nüzi fuchou lun, 25 juin 1907, Tianyi $2: 49-81$.

《Manuscrit du discours à l'occasion de la mort de M. Chen Bufu » 陳君不浮追悼會演説 稿Chenjun bufu zhuidaohui yanshuo gao, 10 juillet 1907, Tianyi $3:$ 549-550.

« Note ajoutée au texte de Liu Shipei, À propos de l'égalité de force [de travail] parmi les hommes »附人類均力説 Fu renlei junli shuo, 10 juillet 1907, Tianyi 3 : 92-93.

《 Note à la lettre de Kotoku Shushui » 幸德秋水來函 Xingde qiushui laihan, 10 juillet 1907, Tianyi 3 : 347-348.

《À propos de la question du travail des femmes » 論女子勞動問題 Lun nüzi laodong wenti, 10 août 1907, Tianyi 5 : 108-120. [Sous le pseudonyme Wei Gong 畏公]

《La question de la libération des femmes » 女子解放問題 Nüzi jiefang wenti, septembre 1907, Tianyi $7: 133-142$.

《Des réussites et échecs de la révolution raciale et de la révolution anarchiste 》論種族 革命與無政府革命之得失 Lun zhongzu geming yu wuzhengfu geming zhi deshi, $1^{\mathrm{er}}$ septembre 1907, Tianyi $6: 120$-133 [Article co-écrit par Liu Shipei, sous le pseudonyme Shenshu 申叔, et He-Yin Zhen].

《Postface aux poèmes de Qiu Jin » 秋瑾詩歌後序 Qiu Jin shige houxu, 15 septembre 1907, Tianyi 7.

《Ce que les femmes devraient savoir à propos du communisme »論女子當知共產主義 Lun nüzi dangzhi gongchan zhuyi, 30 octobre 1907, Tianyi 8-9-10 (compilation) :

167-169.

《De l'antimilitarisme des femmes » 女子非軍備主義論 Nüzi feijunbei zhuyi lun, 30 novembre 1907, Tianyi 11 : 183-189.

《Révolution économique et révolution des femmes » 緇濟革命與女子革命 Jingji geming yu nuzi geming, 30 décembre 1907, Tianyi 13 : 197-205.

《À propos de la cruauté endurée par les femmes chinoises »論中國女子所受之參賭 Lun zhongguo nüzi suoshou zhi candu, 15 janvier 1908, Tianyi 15 : 217-222.

《Lettre de He Zhen en provenance de Zhenjiang » 何震女史由鎮江來函 He Zhen nüshi you Zhenjiang laihan, 8 août 1908, Hengbao 10 : 784-785.

Autres : 
HE-YIN a. « Annonce concernant le journal de la Justice Naturelle » 天義報廣告 Tianyi bao guanggao, juillet 1907, Nüzi shijie 6. Accédé dans : Xia, Xiaohong (éd). 2015. Zhongguo jindai sixiang jia wenku : Jin Tianhe, Lü Bicheng, Qiu Jin, He Zhen juan, Pékin : Zhongguo renmin daxue chuban she : 137-138.

HE-YIN b. « Retranscription des discussions lors de la première réunion de l'Association de lecture et d'étude du socialisme » 社會主義講習會第一次開會記事 Shehui zhuyi jiangxi hui di yi xi kaihui jishi, Tianyi 6, $1^{\mathrm{er}}$ septembre 1907. Accédé dans : WAN Shiguo \& LIU Lydia. 2010. Tianyi, Hengbao [Réimpressions du Tianyi et du Hengbao]. Pékin : Zhongguo renmin daxue chubanshe, Qingshi yanjiu congshu : 307-310.

Recueils de traduction de textes choisis de He-Yin Zhen, en français et en anglais :

HE-YIN, Zhen. 2018. La Revanche des femmes et autres textes. Toulouse : Éditions de l'Asymétrie.

LIU Lydia, KARL Rebecca, \& KO Dorothy. 2013. The Birth of Chinese Feminism: essential texts in transnational theory. New York : Columbia University Press.

\section{NOTES}

1. Il y a un grand flou autour des textes qui ont été écrits, ou pas, par He-Yin Zhen, qui demande davantage de recherches. La chercheuse Xia Xiaohong a fait l'hypothèse selon laquelle une autre contributrice du journal, sous le pseudonyme Zhi Da 志達, puisse être également un pseudonyme de He-Yin Zhen, ce qui ajouterait une dizaine de textes à ceux laissés par He-Yin Zhen (Xia 2014). Il semble aussi très probable (c'est en tout cas l'hypothèse que l'on reprend ici) que Wei Gong 畏 公 soit un de ses pseudonymes. Liu Jen-Peng suggère aussi que He-Yin Zhen ait pu utiliser le pseudonyme de Liu Shipei pour écrire plusieurs textes (Liu 2017 : 3). L'emploi de pseudonymes était très courant, notamment dans les publications anarchistes, et cette pratique n'est pas spécifique aux femmes.

2. Version anglaise : LIU Lydia, KARL Rebecca \& KO Dorothy. 2013. The Birth of Chinese Feminism: essential texts in transnational theory. New York : Columbia University Press. Version française : HEYIN, Zhen. 2018. La Revanche des femmes et autres textes. Toulouse: Editions de l'Asymétrie, Collection Rimanenti. Contrairement à ce que l'édition indique («traduit du chinois par Pascale Vacher »), la traduction française semble avoir été réalisée à partir de la version anglaise des textes. Ainsi la version française a malheureusement repris les passages erronés ou contestables de cette traduction, ainsi que tous les choix de découpage, de ponctuation, de syntaxe et de vocabulaire (voir la note 14 à ce sujet).

3. Voir à ce propos les articles de Liang Qichao. On pourra penser à l'article «À propos de l'éducation des filles ", 論女學 Lun nüxue, dans le Shiwu bao 時務報 (1897).

4. Cette expression n'est cependant pas spécifique à ses textes, car elle est communément employée par d'autres auteures, par exemple dans le journal de Yan Bin, ou même dans d'autres contributions du journal Tianyi.

5. Nous n'entrons pas ici dans les détails de courants multiples de l'anarchisme. Voir à ce sujet l'ouvrage de Peter Zarrow (1990).

6. Ici on note que les traductions anglaise et française prêtent à confusion; en parlant de «man's rule» ou "[le monde a été] régi par les hommes", elles semblent indiquer la domination masculine, alors, qu'en réalité, cette expression ne contient pas le terme homme au sens de masculin (nan 男) mais le terme homme au sens d'humain (ren 人). En traduisant ainsi, elles modifient le sens de l'expression, qui indique au contraire que l'oppression n'est pas fondée sur 
le genre, mais sur l'ensemble d'un système social hiérarchisé et cadenassé. C'est pourquoi on a choisi de le traduire par «système de contrôle social ». Ce terme a été traduit par Peter Zarrow en anglais par le terme « rulership » qui semble plus proche du sens du terme en chinois (1988).

7. La déesse Nüwa 女娲 est un personnage mythologique, présente dans plusieurs textes anciens, qui aurait façonné les êtres humains, en ayant le pouvoir de fondre la pierre, réparer le ciel, arrêter l'eau et le feu, accomplissant ainsi de grands exploits pour leur venir en aide (Lu 2015 : 7).

8. Il faudrait développer une analyse détaillée du lien entre le ren 仁 confucéen et la conception de l'égalité développée à cette époque. Cela permettrait de nuancer la rupture des intellectuels de la fin des Qing d'avec la tradition lettrée confucéenne.

9. Concernant la traduction de ces expressions, et surtout de nan-nü, l'expansion "des sexes » n'est pas très convaincante, c'est pourquoi on la met entre crochets. Nan-nü fait davantage référence aux « rapports entre hommes et femmes » ici.

10. La caractéristique du mot quan 權 est qu'il peut être traduit, selon les contextes, par «pouvoir» ou par «droit». Dans le texte de He-Yin Zhen, nanquan 男權 est le nom donné au "pouvoir masculin", mais nüquan 女權 est le nom donné aux «droits des femmes ». Stephen Angle (1998) montre que le terme quanli 權利 chez Liu Shipei est une notion dérivée du discours confucéen sur l'éthique et la politique, bien plus qu'un pur "néologisme » qui aurait été transplanté dans la langue chinoise pour traduire le terme «droits " à partir de l'anglais, du français ou de l'allemand. Chez Liu Shipei, quanli est lié à la capacité de chacun de " savoir », et à la capacité de chacun "d'agir selon des principes universels ", étant donné que «le cosmos n'a pas créé de distinctions entre les êtres humains». Ce terme a une dimension éthique forte: puisque nos "capacités morales" sont identiques - ainsi, la valeur morale ou vertu supposée supérieure de tel ou tel individu n'est pas fondée - chacun.e a la capacité, et donc aussi le devoir, de participer au bien de la communauté. Les intérêts du peuple sont légitimes. Stephen Angle avance l'argument que ziyou 自由 dans les écrits de Liu Shipei, pourrait signifier " autodétermination " plutôt que « liberté ", en tirant l'action, et la raison de l'action, de soimême (zi). Au contraire, qiangquan est le pouvoir illégitime des souverains ou des hommes qui s'appuient sur la loyauté de «sujets » sans contrepartie. Ce pouvoir est d'autant plus illégitime qu'il s'appuie non pas sur la réciprocité des responsabilités, mais sur la force ; ce pouvoir n'est pas naturel, ni mérité. Imposant des rôles au peuple, il nie leur droit à l'autodétermination. Cette notion de droits renverrait alors à un pouvoir que l'on peut saisir, auquel on peut avoir accès : c'est un levier qui donne le droit d'agir, « ce qui donne la capacité de ». Lorsque, par exemple, HeYin Zhen parle du «droit de participation politique» (canzheng zhi quan 荟政之權) pour les femmes, il s'agit de la possibilité (pouvoir) de voter ou de représenter les votant·e·s. Ainsi lorsque l'on traduit des termes comme qiangquan ou ziyou, faut-il aussi avoir ce contexte linguistique en tête.

11. Pour une discussion détaillée de la traduction et des débats autour de la traduction, voir Sun (2018).

12. Par la réflexion sur l'archéologie du désir en traduction, il y a ici quelque chose qui nous amène vers une critique du régime hétéronormatif du désir, et de ses pratiques de traduction.

13. A titre d'exemple, on pourra se référer à la catégorie « correspondances » du journal Hengbao, qui contient quarante-quatre lettres de provenances variées, de l'Europe à la Chine.

14. On pense ici aux divers travaux de François Jullien, qui contiennent souvent le programme essentialisant (par le biais de la création de concepts philosophiques qui seraient «essentiellement» chinois) dans leurs titres eux-mêmes, et qui reposent sur la création mystifiée de l'« altérité » chinoise.

15. Je me permets de signaler ici une erreur de traduction dans les versions anglaise et française du texte, à la fin du «Manifeste des femmes» (appelé «Manifeste féministe»). En effet, la traduction de Pascale Vacher (à partir de l'anglais) propose :

«En Chine, certaines personnes pensent que pour atteindre cet objectif, les femmes doivent se 
faire les chantres - même en devançant les hommes - des révolutions raciales, politiques, économiques et autres; elles ne doivent pas se permettre d'être de nouveau à la traîne des hommes. De leur point de vue, la révolution entre les hommes et les femmes doit avancer parallèlement aux révolutions raciales, politiques et économiques. Ces femmes pensent que si elles réussissent, elles pourront établir un véritable régime des "droits des femmes " dans le monde. Si elles échouent, elles périront avec les hommes, pour ne pas leur être de nouveau soumises. Je pense qu'il s'agit d'une étroitesse de vue » (p. 127).

Or, les expressions que nous avons soulignées ici ne sont pas présentes dans le texte chinois; de plus, l'expression ququ zhi shi 區區之視 ne signifie pas « une étroitesse de vue ", mais renvoie à l'opinion de l'auteure elle-même, qui dans une posture rhétorique dit «c'est là mon humble opinion " (le dépréciatif renvoie à elle-même, non à d'autres). Ainsi si nous traduisons cette même portion, nous arrivons à ceci, c'est-à-dire au sens tout à fait opposé :

«Si les femmes en Chine souhaitent lutter pour cela, elles devraient, dans les révolutions raciale (zhongzu), politique et économique, non pas rester derrière les hommes, mais les devancer, car la révolution des sexes (nan-nü) doit être menée conjointement avec les révolutions raciale, politique et économique. Si elles y parviennent, ce serait là la véritable réalisation, dans le monde, des «droits des femmes » (nüquan), mais si elles échouent, il vaudrait mieux choisir de périr tous ensemble, plutôt que de devoir souffrir de l'oppression des hommes à nouveau. C'est là mon humble opinion ».

16. Voir, à propos des luttes féministes récentes, l'ouvrage de Leta Hong Fincher (2018), qui porte sur le mouvement \#metoo en Chine. Quelques réserves cependant sur le titre de l'ouvrage, car l' " éveil féministe " proclamé est heureusement bien antérieur au Xxi ${ }^{\mathrm{e}}$ siècle.

17. Voir l'entretien de Wang Zheng dans le blog Lala Zazhi 拉拉杂志 sur Douban : «Cong nüjie zhong dao nanjie zhong : nanxing zhuti, guozu zhuyi, yu xiandai xing » [De la cloche des femmes à la cloche des hommes : le sujet masculin, le nationalisme et la modernité], en date du 15 janvier 2014, consulté le 15 août 2019 https://site.douban.com/211878/widget/notes/13514029/note/ 326848791/]. Cet article est un dialogue entre la chercheuse Wang Zheng et Liu Hedeng sur le texte de Jin Tianhe qui est justement présent dans le recueil The Birth of Chinese Feminism.

18. Certains d'entre eux seulement sont réédités dans l'anthologie de Li \& Zhang (1975). Mais cette anthologie n'est pas facilement accessible et se trouve seulement dans quelques bibliothèques spécialisées. On ne peut pas non plus l'acheter, contrairement à la réédition du Tianyi et du Hengbao.

19. On a vu que le dernier ouvrage de Leta Hong Ficher (2018), qui porte sur la période contemporaine, a pour sous-titre "l'éveil féministe en Chine»

20. Voir à ce sujet l'introduction de l'ouvrage Féminismes islamiques de Zahra Ali (2012). Voir aussi XIONG (2008) et WANG (1999) pour des exemples historiques de féminismes à la fin des Qing.

21. Ces idées sont reprises par les médias et dans les discours officiels de l'État. Elles ne constituent en réalité que les grands traits des discours antiféministes « habituels » et viennent justifier, avec un certain succès, la répression politique des militantes féministes, tout en attachant une connotation très négative et stigmatisante au terme "féminisme » en Chine. Voir à ce sujet Wu \& Dong (2019). Voir aussi Liu (2008). Les personnes perçues comme «féministes » sont aussi accusées de vouloir «imiter » de façon vulgaire les femmes occidentales ; voir Liu (2020).

22. C'est le cas des recherches sur la communauté $\mathrm{Na}$ ou Mosuo, qui seraient parmi les «dernières » communautés matrilinéaires et matriarcales, et qui semblent avoir attiré une certaine attention dans la recherche et les médias français.

23. Voir Nan Z. Da \& Wang Zheng $2015: 8$, pour de nombreuses références critiques et citations à ce sujet. 


\section{RÉSUMÉS}

Cet article propose la traduction inédite d'extraits de textes et articles de la théoricienne anarchiste et féministe chinoise He-Yin Zhen (1884-v.1920), ainsi que la reconstitution d'une liste complète de ses travaux. La redécouverte, la traduction et la confrontation avec ces textes posent de nombreuses questions. La traduction est-elle déjà une interprétation ? Comment rendre compte de l'histoire des termes et de leurs transformations? Qu'ont ces textes à dire sur l'histoire du féminisme, ses théories et leurs circulations? Que nous enseignent-ils sur la production du savoir sur l'histoire chinoise et les « études chinoises »? La traduction peut-elle être un outil pour les luttes féministes actuelles, ou renforce-t-elle les structures coloniales du savoir?

This article proposes the unpublished translation of excerpts from texts and articles written by the Chinese anarchist and feminist theorist He-Yin Zhen (1884-v.1920), as well as an attempt to rebuild a complete list of her works. Re-discovering and translating these texts does raise many questions. Is translation already interpretation? How can we account for the history of the concepts and words she uses and their transformations throughout time? What do these texts have to say about the history of feminism? What do they teach us about knowledge production on Chinese history or in the area of «Chinese Studies »? Can translation be a tool for the ongoing feminist struggles, or does it reinforce the colonial structures of knowledge?

\section{INDEX}

Thèmes : Recherches

Keywords : feminism, China, Japan, history of ideas, press history, anarchism, translation

Mots-clés : féminisme, Chine, Japon, histoire des idées, histoire de la presse, anarchisme, traduction

\section{AUTEUR}

\section{LÉA BUATOIS}

Titulaire d'un Master en études chinoises de l'ENS de Lyon, elle a effectué ses recherches sur l'histoire du féminisme en Chine, et plus particulièrement l'histoire de la presse féminine et féministe du début du vingtième siècle chinois, à partir du fonds d'archives de l'Academia Sinica (Taipei). Elle a également organisé, avec Touriya Fili-Tullon et Fatima Zohra, une journée d'études sur la circulation des idées féministes en langues chinoise et arabe, au sein du projet FELiCiTE (TRIANGLE, ENS de Lyon). 